\title{
6 Der große Wurf? - Ein analytischer Blick auf die erste Förderrunde im Innovationsfonds
}

\author{
Thilo Zelt
}

\section{Der große Wurf?}

Greift der Patient bei einer medizinischen Frage demnächst zum Tablet und geht nicht mehr zum Arzt? Kann die Nutzung von medizinischen und nicht-medizinischen Daten aus verschiedenen Quellen bald Patienten in konkreten Fallkonstellationen bessere Behandlung ermöglichen? Innovationen, sektorübergreifende Lösungen und vor allem die Digitalisierung können und werden das Gesundheitssystem verändern. Um Impulse für Innovation und neue Lösungen zu setzen, wurde im Juni 2015 die Einführung des Innovationsfonds beschlossen, im April 2016 erging die erste Förderbekanntmachung. Dieser Artikel analysiert die seit Ende 2016 bekannten Ergebnisse der ersten Förderrunde. Leitfrage ist dabei, in wie weit der Fonds seine selbstgesteckten Ziele erreicht.

Die Analyse erfolgt unter Berücksichtigung aller öffentlich verfügbaren Informationen zum Innovationsfonds bis inklusive März 2017. Die Analyse basiert auf einer Auswertung der geförderten Projekte nach den Kategorien Verwendung von digitalen Komponenten; Verwendung im direkten Pflegekontext, Verwendung zur Versorgung ländlicher Regionen. 


\section{Der Innovationsfonds soll die sektorübergreifende Versorgung und Forschung stärken}

Der Bundesgesundheitsminister wünscht sich, dass der Innovationsfonds dazu beitragen wird, dass „digitale Verfahren zur Verbesserung der Patientenversorgung, [...] Mauern einreißen und Brücken zwischen den Versorgungsbereichen bauen“ (FAZ 2017). Die Erwartungen an den Innovationsfonds sind allerdings durchaus unterschiedlich. Der Name des Fonds, der Koalitionsvertrag zwischen CDU, CSU und SPD (Deutschlands Zukunft gestalten) sowie die Aussagen des Bundesgesundheitsministers zielen auf Innovation und Hebung digitaler Potenziale, die der Fonds bewirken soll. Aussagen des Vorsitzenden des gemeinsamen Innovationsausschusses Josef Hecken beim BMC-Diskussionsforum am 14.09.2015 in Berlin zur Ausgestaltung des Innovationsfonds und auch die Förderbekanntmachung zielen jedoch vor allem auf die Überführung von bestehenden (sektorübergreifenden) Lösungen in die Regelversorgung. Konkret möchte der Innovationsfonds gesellschaftliche Herausforderungen lösen (BMC 2016). Vor allem gefördert werden sollen:

- Angebote mit Fokus auf die demografische Entwicklung,

- Behandlungsformen im Versorgungsalltag, die eine sektorenübergreifende Versorgung einschließlich geeigneter Schnittstellen zu Prävention, Rehabilitation und Pflege ermöglichen und

- Lösungen für unterschiedliche Versorgungssituationen in Ballungsräumen, strukturschwachen Regionen und ländlichen Regionen.

Die gesteckten Ziele sind mit Blick auf die aktuelle Situation im deutschen Gesundheitswesen ambitioniert: So nimmt das Gesundheitswesen den vorletzten Platz im Digitalisierungsgrad von 11 Branchen in Deutschland ein (BMWi 2016), und sektorübergreifende bzw. integrierte Versorgung sind noch gering ausgeprägt (ICES Institut 2014). Dem Innovationsfonds stehen 2016 bis 2019 jährlich 300 Millionen Euro zur Verfügung. Davon sollen 225 Millionen in die Förderung neuer Versorgungsformen und 75 Millionen in die Förderung der Versorgungsforschung investiert werden. Im Rahmen der ersten Förderrunde werden nun 29 Projekte zu Neuen Versorgungsformen und 62 Projekte zur Versorgungsforschung gefördert.

\section{Die üblichen Verdächtigen dominieren die vom Innovationsfonds geförderten Projekte}

Insgesamt kommen mehr als zwei Drittel (69 Prozent) der Konsortialführer erfolgreicher Projektanträge von Universitäten. Die Projekte werden entweder direkt von Universitäten (33 Prozent) oder von Universitätskliniken (36 Prozent) geleitet. Daneben wurden geförderte Projekte insbesondere von Krankenkassen (1o Prozent) und privaten Unternehmen (8 Prozent) federführend beantragt. Die Verteilung der Fördernehmer ist dabei nicht verwunderlich: Bereits der Gesetzestext beschreibt, welche Teilnehmer für den Innovationsfonds gewünscht sind: Krankenkassen werden im Bereich Neue Versorgungsformen explizit als Teilnehmer genannt, und Universitäten sollen im Bereich Versorgungsforschung teilnehmen (SGB V, $\$ 92 a)$.

Dem entsprechend werden im Bereich Versorgungsforschung mehr als drei Viertel (78 Prozent) der Projekte von Universitäten (47 Prozent) und Universitätskliniken 
(31 Prozent) koordiniert. Nur in seltenen Fällen wurden Forschungsprojekte von anderen Akteuren wie privaten Unternehmen (5 Prozent) oder Krankenkassen (3 Prozent) initiiert. Bei den Neuen Versorgungsformen dominieren ebenfalls die Universitäten (3 Prozent) und Universitätskliniken (48 Prozent). Dabei treten hier aber Krankenkassen und private Unternehmen häufiger als Antragssteller auf. 24 Prozent der Projekte werden durch Krankenkassen und 14 Prozent durch private Unternehmen initiiert.

Während die Mehrzahl der Hauptantragssteller aus dem universitären Kontext kommt, werden als weitere Projektpartner insbesondere Krankenkassen (51 Prozent) in die Projekte eingebunden. Aber auch private Unternehmen (27 Prozent) und nichtuniversitäre Krankenhäuser sowie andere Gesundheitseinrichtungen (2o Prozent) nehmen teil.

\section{Digitalisierung ist das zentrale Innovations-Thema}

Mit 37 Prozent beschäftigt sich mehr als ein Drittel der geförderten Projekte mit Digitalisierungsthemen. Dies spricht dafür, dass der Innovationsfonds eine Grundlage für eine weitergehende Digitalisierung des deutschen Gesundheitssystems schaffen kann. Digitalisierungsprojekte zielen dabei stark auf die Verbesserung von Organisationsabläufen. 6o Prozent der Projekte, die prozessuale oder organisatorische Verbesserungen im Gesundheitswesen adressieren oder erforschen, verfolgen einen Digitalisierungsansatz.

Die Digitalisierungslösungen zielen aber selten auf die gesellschaftlichen Herausforderungen, die der Innovationsfonds lösen will: Nur 4 Projekte mit Digitalisierungsbezug adressieren den demografischen Wandel, 2 zielen auf den Pflegesektor und 6 Projekte auf die Verbesserung der Versorgung in ländlichen Regionen.

\section{Projekte im Bereich Neue Versorgungsformen setzen auf digitale lösungen - allerdings wenig Fokus auf die gesellschaftlichen Ziele des Fonds}

Im Bereich Neue Versorgungsformen werden mit der ersten Förderwelle 29 Projekte gefördert. Die durchschnittliche Fördersumme beträgt ca. 7,8 Millionen Euro Dabei ergibt sich eine recht ausgeglichene Verteilung auf die gesetzten Themenschwerpunkte:

- Versorgungsmodelle in strukturschwachen oder ländlichen Gebieten (14 Prozent)

- Modellprojekte zur Arzneimitteltherapie sowie Arzneimitteltherapiesicherheit (1o Prozent)

- Versorgungsmodelle unter Nutzung von Telemedizin, Telematik und E-Health (21 Prozent)

- Versorgungsmodelle für spezielle Patientengruppen (38 Prozent)

5 Projekte sind themenoffen vergeben worden. Insgesamt zeigt sich, dass knapp 70 Prozent, also 20 der 29 Projekte im Bereich Neue Versorgungsformen, Potenziale der Digitalisierung heben wollen. In knapp der Hälfte aller geförderten Projekte wer- 
den dabei telemedizinische Anwendungen erprobt. Digitale Arbeits- \& Verwaltungsunterstützung und E-Learning kommen in jeweils 3 Projekten zum Einsatz. Nur 9 Projekte verzichten komplett auf digitale Anwendungen.

Die übergeordneten Ziele des Innovationsfonds, die durch die Ausschreibung definiert wurden, werden jedoch durch die Projekte aus der ersten Förderwelle nur begrenzt adressiert:

- Immerhin 6 der 29 Projekte, also knapp 20 Prozent, haben einen expliziten Bezug zum Bereich Förderung der Gesundheitsversorgung in ländlichen Räumen.

- Nur 14 Prozent der 29 Projekte versuchen medizinische Herausforderungen des demografischen Wandels zu lösen.

- Das Thema Pflege, relevant für die sektorübergreifenden Versorgung und dem demografischen Wandel, wird nur von 2 Projekten aufgegriffen.

\section{Projekte im Bereich Versorgungsforschung sind weniger digital orientiert und zielen verstärkt auf Patientensicherheit}

Im Bereich Versorgungsforschung werden insgesamt 62 Projekte gefördert. Die durchschnittliche Fördersumme liegt bei ca. 1,2 Millionen Euro. Die Forschungsprojekte beziehen sich auf einen der folgenden Themenschwerpunkte oder konnten themenoffen eingereicht werden:

- Weiterentwicklung der Qualitätssicherung und/oder Patientensicherheit in der Versorgung (24 Prozent)

v Verbesserung von Instrumenten zur Messung von Lebensqualität für bestimmte Patientengruppen (8 Prozent)

- innovative Konzepte patientenorientierter Pflege (3 Prozent)

- Verbesserung der Bedarfsgerechtigkeit und/oder Wirtschaftlichkeit der GKVVersorgung (16 Prozent)

- Ursachen, Umfang und Auswirkungen administrativer und bürokratischer Anforderungen im Gesundheitswesen auf die Patientenversorgung sowie Entwicklung geeigneter Lösungsansätze (2 Prozent)

- Einsatz und Verknüpfung von Routinedaten zur Verbesserung der Versorgung (19 Prozent)

- Evaluation von Selektivverträgen/Evaluation der SAPV-Richtline des G-BA (11 Prozent)

v themenoffen (16 Prozent)

Insgesamt verfolgen hier 14 Projekte (33 Prozent) einen Digitalisierungsansatz. Hauptfokus der Projekte sind bei diesen datengestützte Analyse und Big-Data-Anwendungen (8 Projekte). So entwickelt beispielsweise ein Projekt Methoden zur Nutzung von Routinedaten für ein sektorenübergreifendes Entlassungsmanagement.

Auch im Bereich der Versorgungsforschung zeigt sich, dass die gesellschaftlichen Ziele des Fonds nur begrenzt adressiert werden:

- Der demografische Wandel wird von knapp 20 Prozent der Projekte adressiert,

- nur knapp 10 Prozent der Forschungsprojekte adressieren das Thema Pflege und

- kein Projekt aus dem Bereich Versorgungsforschung adressiert das Thema Gesundheitsversorgung in ländlichen Regionen. 


\section{Zwischenfazit}

Insgesamt zeigt sich, dass der Innovationsfonds mit der ersten Förderrunde durchaus innovative Ansätze fördert und dass Digitalisierungslösungen breit zum Zuge kommen. Dabei ist allerdings fraglich, ob die definierten gesellschaftlichen Ziele hinreichend adressiert werden. Auch die Vielfalt an digitalen Innovation könnte größer sein. Digitale Anwendungen sind in der ersten Förderwelle häufig auf Einzellösungen fokussiert und wenig sektorübergreifend angelegt. Die Antragssteller und die Teilnehmer der Anträge sind überwiegend alte Bekannte, insbesondere Universitäten und Krankenkassen leiten und betreiben die Projekte.

Damit der Innovationsfonds die hohen Erwartungen, die an ihn gestellt worden sind, erfüllen kann, sollten auf Basis der Analyse der Projekte der ersten Förderrunde folgende Impulse berücksichtigt werden:

1. Nicht nur die klassischen Akteure unterstützen - Start-ups und die Privatwirtschaft insgesamt stärker zur Teilnahme an Projekten ermutigen,

2. Raum für mehr innovative Digitalisierungslösungen schaffen - Projekte sollten gezielt neue digitale Lösungen adressieren,

3. Digitalisierungslösungen sollen die Versorgung der Patientinnen und Patienten unterstützen - nicht nur die Verbesserung der Prozesse,

4. gesellschaftliche Herausforderungen stärker in den Fokus stellen - auch bei digitalen Lösungen und

5. themenoffene Beiträge stärker steuern und mehr auf die gesellschaftlichen Herausforderungen hin entwickeln.

\section{Literatur}

Bundesministerium für Gesundheit (BMG) 2016 Geförderte Projekte des Innovationsausschusses zur Förderbekanntmachung Neue Versorgungsformen vom 8. April 2016. URL: https://innovationsfonds.g-ba.de/ downloads/media/47/Neue-Versorgungsformen_Uebersicht-gefoerderte-Projekte-2016.pdf (abgerufen am 21.07.2017)

Bundesministerium für Wirtschaft und Energie (2016) Monitoring-Report Wirtschaft DIGITAL 2016. URL: https:// www.bmwi.de/Redaktion/DE/Publikationen/Digitale-Welt/monitoring-report-wirtschaft-digital-2016. html (abgerufen am 21.07.2017)

Frankfurter Allgemeine Zeitung (FAZ) (2017) Mit Datenschutz zum Datenschatz. Was die Digitalisierung des Gesundheitswesens leisten muss. Von Hermann Gröhe, 09.01.2017, Nr. 7, S. 18

IGES Institut (2014) Monitoring Integrierte Versorgung Rahmenbedingungen der IV verbessern. Dritte Erhebung - Expertenbefragung 2014 im Auftrag der Deutschen Gesellschaft für Integrierte Versorgung im Gesundheitswesen (DGIV). Studienbericht des IGES Instituts Berlin, August 2014 